\title{
The Specialty of Geren in Huarong Dialect of Hunan Province
}

\author{
Cai Li \\ College of Chinese Language and Culture, Jinan University, Guangzhou, Guangdong, China
}

\begin{abstract}
In Huarong dialect of Hunan province, "geren" has two usages. One is "geren 1", whose meaning and usage is just like the Mandarin reflexive " $z i j i "$. In additon, "geren" has a special usage—commentary adverb "geren ${ }_{2}$. "Geren ${ }_{2}$ " is a subjective marker of discourse whose function is to strengthen modality or stress certain situation.
\end{abstract}

Keywords-Geren; Huarong dialect of Hunan province; pronoun; oneself; commentary adverb; subjective marker

\section{湖南华容话中“各人”的特殊用法}

\author{
蔡丽 \\ 暨南大学华文学院, 广州, 广东, 中国
}

摘 要 湖南华容话中“各人”有两种用法。“各人 1 为代词, 与普通话的反身代词“自己”意义及用法基本相当。“各人 $2_{2}$ 用法特殊, 为 评注性副词, 充当话语中主观标记, 起加强某种语气或突出某种情状的作用。

关键词 各人; 湖南华容话; 代词; 自己; 评注性副词; 主观标记

\section{1. 引言}

湖南省华容县地处长江中游南岸、洞庭湖西部, 位于 湖南省最北部, 岳阳市西境, 今属岳阳市。华容县所辖部 分乡镇的语言与城关镇存在较大差异。本文所称的湖南华 容话是指湖南省岳阳市华容县城, 即城关镇本地人所说的 话。(1)

在现代汉语普通话及许多方言中都有“各人”这个代 词。《汉语大词典》在该词条下共列出两个义项: 1 . 各个人; 每个人。2. 自己。在普通话中第一个义项比较常用。而在西 南官话区的一些方言点, “各人”的意思及用法与普通话中 的反身代词“自己”相当, 周作明、马友平 (2006) 的研究 表明四川方言中的这个代词与 “自己”基本一致。在华容话 中, “各人”用作代词时, 主要表示“自己” (记为“各人 ”); 除此之外, “各人”还有一种不具任何指代作用的特殊用法 一“各人 2 ”, 本文将主要论述这种用法。

\section{2. “各人 ${ }_{2}$ ”的语法意义及句法特征}

“各人 2 ”的基本语法意义是加强某种语气, 突显某种情
状。在不同语境及上下文中, “各人 $2_{2}$ ”的具体语法意义略有 不同。具体情况如下:

\section{1 表示强调}

(1) 电话各人紧他搞烂哒。电话让他弄坏了。

(2) 你的脚各人烂得咯好样子哒, 还说冒得事。你的 脚都烂成这样了, 还说没事。

（3）饭弄好吗? 我各人会饿死去。饭做好了吗? 我快 饿死了。

（4）天佬又各人变冷哒。天气又变冷了。

(5) 咯几年他各人搞得蛮好哒。这几年他搞得很好了。

上述几例中的“各人”都出现在主谓之间, 它们没有具 体的词汇意义, 在普通话中找不到与之完全对应的词语, 其作用是强调、突出其后出现的某种情况, 使表达带有明 显的主观色彩。

附注: (1)湖南华容话为本文作者的母语, 全文例句曾在母语区进行过实地验证。书面记录时主要根据华容话的标准读音用普通话中发 音近似的字转写, 如有偏颇, 概由作者本人负责。

(2)董秀芳（2003）采用“复合反身代词”这一概念指称“代词+自己”这种组合, 本文用此名称来称呼“代词/名词+自己”这种组合。 


\section{2 出乎意料}

（6）到只几天冒看倒他, 他就各人变得咯好样子哒。 才几天没见到他，他居然变成这个样子了。

(7) 他们都各人考起大学哒。他们居然都考上大学了。

（8）发生哒咯么大的事, 他各人还不晓得。发生了这 么大的事，他竟然还不知道。

（9）我到只骂哒他两句, 他就各人跑哦哒。我才骂了 他两向, 他竟然就离家出走了。

（10）到只五点钟, 就各人黑哦哒。才五点钟, 天就 黑了。

例（5）～（10）的“各人”也是出现在单句或复句后一 分句的谓语之前, 表达一种出乎意料、惊讶的语气。其表 达效果与普通话的“竟然”“居然”近似, 但这些词在普通话中 一般都出现于其他副词之前。在华容话中, 如果谓语部分 有其他副词, “各人 2 ”可位于副词之后, 也可以位于副词之 前, 其出现的位置依说话人主观上要突出的内容而定, “各 人 2 ”往往紧邻说话人要突出的信息或情况。如果其他副词 所表达的信息也是说话人要强调的内容, “各人 2 ”就可以位 于副词之前, 如例 (8); 而当其他副词所表达的信息并非 说话人想突出的部分时, 或其他副词本身己经通过其他方 式得以突显时, “各人 2 ”就可以位于副词之后, 如例 (6) (9) （10）中“才......就......”有突出作用, “各人 2 ”就放在副 词之后、谓语动词之前。例（7）“各人 2 ”位于“都”之后, 表示对他们“考起大学”这件事感到意外, 此时“都”不能重 读; 如果“都”重读, 就起强调“他们全都考起大学”这一情况 的作用。

\section{3 突出不满的意味}

（11）他到只搞哒一下儿就各人跑哦哒, 好倒我一个 人在咯里一直搞得咯么时候。他才千了一会儿就跑了, 让 我一个人在这里一直干到现在。

（12）他每个月到只交哒八百块钱就各人随唔支都不 管哒。他每个月才交了八百块钱就什么都不管了。

（13）读哒几年书, 他各人随哪个都看不起哒。读了 几年书，他就什么人都看不起了。

（14）他只看哒半个小时书就各人玩去哒。他只看了 半个小时书就去玩了。

（15）去哒咯么多次, 他各人还不认得路。去了这么 多次, 他还不认识路。

例 (11) (13) 的命题本身含有不满的态度, “各人 2”在句中突出了说话人对其后出现的某种情况的不满的意
味。例（14）（15）命题本身并没有什么消极色彩, 但是用 了“各人 2 ”之后, 就传递了说话人对这种情况不满的情绪。

2.4 突出建议

（16）你只各人自己搞个店子, 不跟他一起搞哒。你 不如自己开个店, 别跟他一起千了。

（17）你各人跟他请个老师到屋里来教他还好些。你 给他请个老师到家里来教他更好。

（18）你各人去看电视, 不到咯里吵我啰。你去看电 视吧，别在这儿吵影响我。

（19）咯么晚哒, 又在的下雨, 索性各人明儿去算哒。 这么晚了, 又在下雨, 干脆明天去算了。

上述各例中的“各人 2 ”都出现在表示某种建议的句子中, 突出说话人提出的某种建议。普通话中在表达建议时, 为 了区别于命令语气, 往往会加上一些表达委婉语气的词语, 如“不如”、“吧”等。而华容话中“各人 2 ”负载了建议的语气, 因此不需要再用别的词语来缓和语气。例如 (18), 如果去 掉“各人 2 ”, “你去看电视”是一种命令的语气, 而加上“各人 2 ”后, 就表示一种建议了。

\section{5 表示只管}

(20) 你们各人先吃啰, 不兴等得我。你们先吃, 不 用等我。

（21）你们各人放肆吃, 吃哦哒又买。你们尽量吃吧, 吃完了又买。

（22）外路在的玩龙, 我们一下看热闹去哒, 他各人 看他的书, 一滴嘎嘎都冒分心。外面在舞龙, 我们全去看 热闹了，他只管看书，一点都没分心。

（23）你的孩儿交得他只各人放一百个心。你的孩子 交给他就尽管放心吧。

例（20）～（23）的“各人 2”表达的是“只管、尽管”的意 思。其中例（20）（21）似乎也可以理解为建议, 但此处, 说话人的本意并非是提出建议, 而是希望听话人毫无顾忌 地做某事。

\section{6 小结}

根据上述例句可以归纳出“各人 2 ”使用时的句法特征: （1）不单独出现在句首，一般位于主谓之间的状语位置。 (2) “各人 2 ”前的主语可以是人, 也可以是物; 可以是单 数, 也可以是复数; 主语可以是显性的, 也可以是潜主语, 如例（10）（19）。（3）不能充当主语、定语和宾语。(4) 
如果谓语前有其他副词作状语, “各人 2 ”一般位于副词之 后, 有时也可位于副词之前, 视说话人的主观表达需要而 定。(5) 当谓语动词前有否定词或介词短语时, “各人 2 ” 只 能位于否定词或介词短语之前。

就“各人 2 ”出现的句法环境而言, 接近副词的句法特 点。根据其句法性质及语法意义, 可将其视为评注性副词。

Lyons（1977）认为“主观性是指语言的这样一种特性, 说话人在说出一段话的同时表明自己对这段话的立场、态 度和感情。”(转引自沈家煊, 2001) 鲁川 (2003) 认为语 言中的主观信息表现为主观因素和主观标记, “主观标记” 是话语中显露在客观信息词语 (实词) 之外的专门表达言 者的主观意图、主观情绪和主观态度的语意标记 (虚词), 包括“语气标记”和“情态标记”。从“各人 2 ”这个词的表义特 征及实际表达效果来看, 我们认为它是一种表现语言“主观 性”的标记, 蕴含了说话人的某种“认识”, 说话人一旦用上 这个词, 就表明了自己对所说客观内容的某种主观立场、 主观态度或主观情绪, 从而在话语中留下鲜明的主观印记。 如果说话人要客观地叙述某种情况是不能加“各人 2 ”的。“各 人 ” 是体现说话者基于自身的主观认识而对事情产生的主 观态度或情绪的, 属于一种“情态标记”。

\section{3. 华容话中“各人 2 ”形成过程的推测}

根据“各人 2 ”出现的句法环境, 我们推测华容话中“各 人 2 ”这一特殊用法最初应是由居于主语位置的复合反身代 词 ${ }^{2}$ 虚化而形成的。

在主语位置的复合反身代词中的“各人”一开始是代 词, 表示“自己”。Kaburaki（1973）曾指出日语中反身代词 是一种主观性的表达, 董秀芳认为汉语中的反身代词“自 己” 也是一个主观性表达, 叙述者是站在反身代词的先行语 所代表的人物的立场来观察事件的。（董秀芳, 2003）华 容话中的“各人 1 ”与普通话“自己”相当, 我们认为它也是一 种主观性的表达。

由于“各人 1 ”经常出现在主语位置的复合反身代词这 种句法环境中, 语义逐渐发生偏移, 复合反身代词的指代 义逐渐向前面的先行语倾斜, “各人”的指代功能开始弱化, 但其主观性特征得以保留, 同时复合反身代词的强调意味 附着在“各人”之上, 从而使其衍生出加强某种语气的表达 效果。此时, “各人”可重新分析为评注性副词—“各人 2 ”。

当这种表义功能稳定下来之后, “各人 2 ”在句法上就开 始获得一定的灵活性, 此时, “各人 2 ”不必再拘限在主语之 后、与主语紧邻的位置, 而可以自由出现在句中任何情态 成分可以出现的位置。这一语法化过程可描写如下:

（24）你各人吃, 不兴管得我。你自己吃, 不用管我。 （主语位置的复合反身代词, “各人”表示“自己”)
“各人”开始虚化)

$\downarrow$ (向法形式不变, 语义发生倾斜,

你各人吃, 不兴管得我。你吃, 不用管我。（“各 人”不表示“自己”)

$\downarrow$ (“各人”完全虚化, 开始与先行

语分离）

你只各人吃, 不兴管得我。你只管吃, 不用管

我。（“各人”加强“只管”语气）

总之, 华容话中的“各人 2 ”是“各人 1 ”虚化而形成的, 但在语法化的同时, “各人”仍然保留了它作为实词的基本 用法——“各人 1 , 因此, 在实际语言表达中就可能存在歧 义现象。例如:

（25）她各人把锁都换哦哒。

例（25）是一个有歧义的句子, 可以表达两种意思: 一种是“她自己把锁都换了。”“各人”在句中为代词, 与先行 语“她”构成同位短语, 强调施事主体是“她”, 而不是别人。 第二种意思是“她竟然把锁都换了。”“各人”在句中为评注性 副词, 起强调“把锁换了”这一情况的作用。在语音上, “各 人 2 ”里的“人”读得比较轻、比较快, 一定不能重读; 而“各 人 ”里的“人”在强调时可以重读。

\section{4. 结论}

张谊生（2000）认为, 汉语历史语法里副词多由名词、 动词、形容词这三类实词虚化而来; 诱发汉语实词副词化 的句法结构关系主要包括动宾结构、连动结构和联合结构, 其中联合结构指谓词性联合结构。本文的研究表明华容话 里的评注性副词“各人 2”来源于代词, 而实现副词化的结构 为体词性联合结构。这一语言事实说明现代汉语方言中的 副词有着与普通话不完全相同的来源。

按鲁川 (2003) 对情态范畴的分类, 普通话中的情态 标记基本上是专职的, 每个标记只能表达一种情态, 但华 容话中“各人 2 ”既可表判断 (强调、出乎意料) 又可表评议 (不满、建议、只管), 是一种跨类的多功能的情态标记。

\section{参考文献(References)}

[1] Dong Xiufang, the Pronouns, Reflexive Pronouns and Zero Form on the Attributive Position, Research and Exploration of Grammar,vol.12,2003.

[2] Lu Chuan, The Subjective Informations of Languages and the Modal Markers of Chinese, Research and Exploration of Grammar,vol.12, 2003.

[3] Luo Zhufeng, Grand Chinese Dictionary, the Press of the Great Chinese Dictionary,1989.

[4] Shen Jiaxuan, A Survey of Studies on Subjectivity and 
Subjectivisation,Foreign Language Teaching and Research, vol.4,2001.

[5] Zhang Yisheng,A Study on the Adverbs in Modern Chinese,
Xuelin Press,2000.

[6] Zhou Zuoping, Ma Youming. A Study on Geren in Sichuan Dialect, Social Sciences of Chongqing , vol.11,2006. 